\title{
SIMULATION OF THE RECRYSTALLIZATION TEXTURES OF AL-ALLOYS ON THE BASIS OF NUCLEATION AND GROWTH PROBABILITY OF THE VARIOUS TEXTURES COMPONENTS
}

\author{
O. ENGLER
}

\author{
Institut für Metallkunde und Metallphysik, RWTH Aachen, Kopernikusstr. 14, \\ D-52056 Aachen, Germany
}

(Received 18 April 1996)

\begin{abstract}
The recrystallization textures of cold rolled Al-alloys are simulated under the assumption of a growth selection of the typical recrystallization texture components out of a limited spectrum of preferentially formed nucleus orientations. The probability of nucleation is derived from the distribution of the potential nucleus orientations which was determined by EBSD local texture analysis for the most important nucleation sites in cold rolled commercial Al-alloys, i.e. Cube-bands, grain boundaries and second phase particles. If several nucleation sites are active simultaneously, the total nucleation probability is composed of the respective contributions of each nucleation site. The growth probability is derived from a $40^{\circ}<111>$ transformation of the rolling texture. The resulting recrystallization textures are simulated by multiplying these two probability functions, i.e. the ODF of the nucleus orientations with the ODF of the $40^{\circ}<111>$-transformed rolling texture.
\end{abstract}

KEY WORDS: Recrystallization texture simulation, transformation texture, recrystallization, oriented nucleation, oriented growth, EBSD, Al-alloys.

\section{INTRODUCTION}

The changes in the crystallographic texture during the recrystallization of a deformed metallic material are generally interpreted in terms of two theories, which are based on the two fundamental mechanisms of recrystallization, the formation of new undeformed nuclei and their subsequent growth into the surrounding matrix. (i) In the case of oriented nucleation it is assumed that the preferred formation of special orientations determines the final recrystallization texture. In deformed Al-alloys, recrystallization nucleation generally involves enhanced subgrain growth in the vicinity of structural heterogeneities, where substantially larger local misorientations are built up during deformation than in the homogeneously deformed matrix (e.g. Doherty, 1978). In heavily rolled commercial Al-alloys, mainly three different nucleation sites are of importance, namely Cube-bands, grain boundaries and the deformation zones around large particles (e.g. Juul Jensen, Hansen and Humphreys, 1985, Engler, Vatne and Nes, 1996). For each of these three characteristic nucleation sites specific distributions of nucleus orientations $f(g)^{\text {nucl }}$ have been identified, which in case of recrystallization being solely controlled by oriented nucleation will dominate the recrystallization textures.

(ii) In the case of oriented growth it is assumed that starting from a broad spectrum of nucleus orientations those with the best growth conditions with respect to the 
deformed matrix grow fastest and, therefore, dominate the recrystallization texture. In $\mathrm{Al}$, a $40^{\circ}<111>$ orientation relationship between the nucleus and its surroundings was observed to lead to the highest growth rates (Lücke, 1974), and hence, it can be assumed that the growth probability $f(g)^{\text {grow }}$ corresponds to the so called transformation texture, which can be generated by a numerical $40^{\circ}$-rotation of the deformation texture $f(g)^{\text {def }}$ around all possible $<111>$-axes. If the recrystallization of a sample is totally governed by a selection of grains with a $40^{\circ}<111>$ orientation relationship to the deformation texture, the resulting recrystallization textures can be simulated by this texture transformation (e.g. Pospiech and Lücke, 1979, Lücke and Engler, 1990, 1992, Köhler et al., 1992).

However, these two theories for recrystallization texture interpretation only represent limiting cases and a simulation of recrystallization textures solely based on either of these two theories mostly fails. Instead, a combination of both theories in the sense of growth selection out of a limited spectrum of preferentially formed nucleus orientations must be taken into consideration, which is able to account for the recrystallization textures of most Al-alloys (Lücke and Engler, 1992, Engler, 1996). In that instance, it appears reasonable to multiply the probability function $f(g)^{\text {nucl }}$ of the occurrence of the nucleus orientations and the probability function $f(g)^{\text {grow }}$ of their growth to simulate the probability of the orientations to arise in the final recrystallization texture $f(g)^{\text {sim }}$ (Figure 1). In the literature, there are several attempts to simulate recrystallization textures based on this assumption (e.g. Bunge and Plege, 1987, Jonas, Tóth and Urabe, 1994, Engler, Hirsch and Lücke, 1995, Köhler and Bunge, 1995). The main drawback of this approach, however, is to get reliable data on the nucleation probability functions.

Nowadays, single grain orientation measurements by means of Electron Back Scattering Diffraction (EBSD) are able to yield quantitative information about the orientations at the various nucleation sites. In the present study, the distributions of

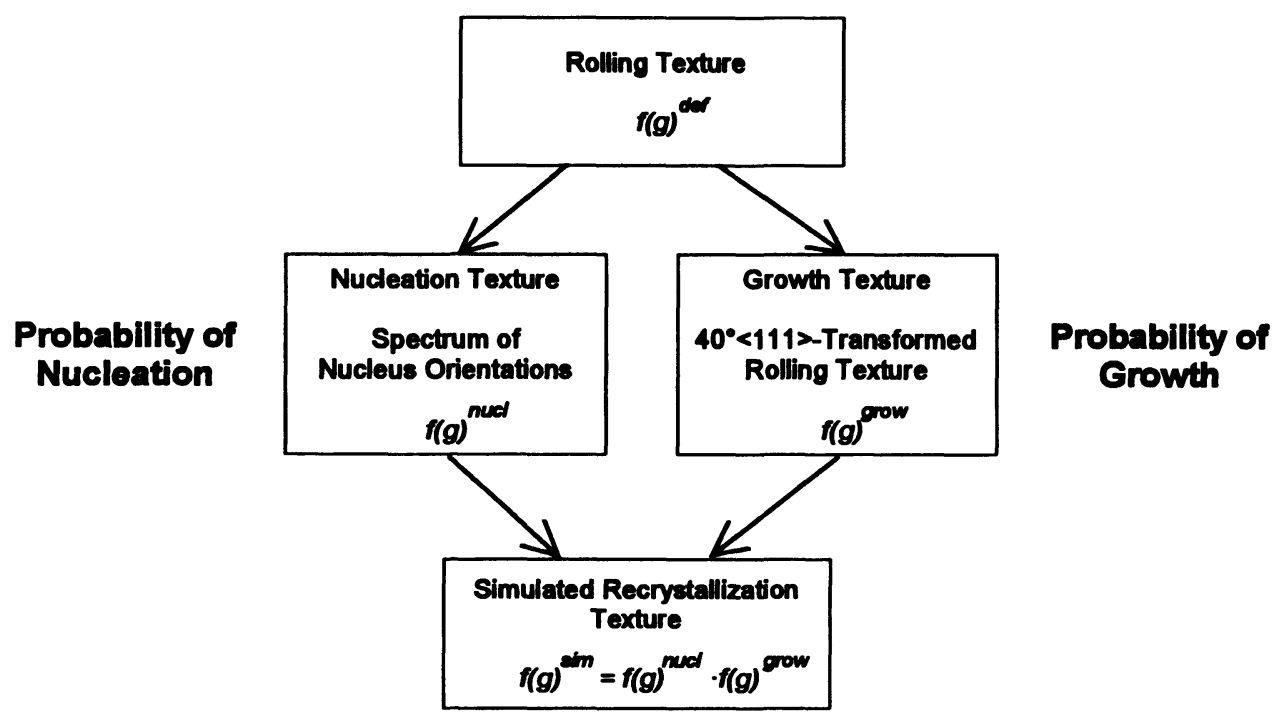

Figure 1 Scheme of the model. 
the nucleus orientations provided at the three most important nucleation sites active in heavily cold rolled commercial Al-alloys, i.e. the Cube-bands, grain boundaries and particles, were directly determined by means of EBSD, and the ODFs computed from these data were assumed to correspond to the respective nucleation probabilities $f(g)^{\text {nucl }}$. The resulting ODFs in combination with the $40^{\circ}<111>$ transformed rolling textures, which consider the growth probability $f(g)^{\text {grow }}$, were finally utilized to simulate the recrystallization textures of different Al-alloys.

\section{THE MODEL}

The distribution of the crystallographic orientations $g$ in a sample, i.e. its crystallographic texture, is given by the orientation distribution function (ODF) $f(g)$ which can be presented in terms of a series expansion to (symmetrized) spherical harmonic functions $T_{l}^{\mu \nu}$ with the coefficients $C_{l}^{\mu \nu}$ (Bunge, 1982):

$$
f(g)=\sum_{l=1}^{l_{\max }} \sum_{\mu=1}^{M(l)} \sum_{v=1}^{N(l)} C_{l}^{\mu \nu} \cdot T_{l}^{\mu \nu}(\mathrm{g})
$$

In order to determine the nucleation probability in a given sample state, the frequency of the nucleus orientation, i.e. their ODF $f(g)^{\text {nucl }}$, has to be determined. For that purpose, the orientations of a multitude of nuclei or first recrystallized grains in partially recrystallized sample stages were measured by means of Electron Back Scatter Diffraction (EBSD) in a SEM (Engler and Gottstein, 1992), as discussed in more detail elsewhere (Engler, Yang and Kong, 1996, Engler, 1996). From the resulting microtexture data, ODFs were computed by associating each single orientation $i$ with a Gauss type peak in Euler angle space with a (half) scatter width $\psi_{0}=8^{\circ}$. For a texture consisting of $N$ individual orientations the ODF can be determined by superposition of the corresponding $N$ Gauss-peaks (Pospiech and Lücke, 1975). The C-coefficients of the series expansion method can then easily be calculated by:

$$
C_{l}^{\mu \nu}=\frac{\exp \left(-\frac{l^{2} \psi_{0}^{2}}{4}\right)-\exp \left(-\frac{(l+1)^{2} \psi_{0}^{2}}{4}\right)}{1-\exp \left(-\frac{\psi_{0}^{2}}{4}\right)} \cdot \sum_{i=1}^{N} M_{i} \cdot T_{l}^{\mu \nu}\left(g_{i}\right)
$$

(with $l_{\max }=22$ ). For analysis of the spectrum of nucleus orientations it is most reasonable to assume that the orientation of each grain $i$ contributes to the final nucleation texture with the same volume fraction $M_{i}=1 / N$ without considering its size. The resulting ODFs were assumed to correspond to the nucleation probability $f(g)^{\text {nucl }}$.

With regard to the preferred growth of grains with a $40^{\circ}<111>$-orientation relationship to the deformed matrix, the growth probability $f(g)^{\text {grow }}$ is assumed to correspond to the $40^{\circ}<111>$-transformed rolling texture. Thus, the rolling texture $f(g)^{\text {def }}$ had to be transformed according to:

$$
f(g)^{\text {trans }}=\frac{1}{8} \sum_{i=1}^{8} f\left(\Delta g_{i} \cdot g\right)
$$

where the $\Delta g_{i}$ denote the eight different $40^{\circ}<111>$-rotations. 
The ODFs which describe the textures of the rolled sheets were computed from four pole figures $(\{111\},\{002\},\{022\},\{113\})$ measured by standard X-ray diffraction techniques by means of the series expansion method with $l_{\max }=22$. From the Ccoefficients of the rolling texture (Eq. (1)), the $\hat{C}$-coefficients of the transformation texture can be calculated using the following transformation law (Bunge, 1982):

$$
\hat{C}_{l}^{\mu \nu}=\frac{1}{2 l+1} \sum_{\mu=1}^{M(l)} C_{l}^{\mu \nu} \cdot W_{l}^{\mu \mu}
$$

where $W$ is the transformation law function. If $W$ consists only of one orientation relationship $\Delta g$ including its symmetrical variants, then $W$ is defined as follows:

$$
W_{l}^{\mu \mu}=(2 l+1) T_{l}^{\mu \mu}(\Delta g)
$$

In order to take both influences into account, i.e. a growth selection out of a limited spectrum of nucleus orientations, the probability densities of the two ODFs $f(g)^{\text {nucl }}$ and $f(g)^{\text {grow }}$ must be multiplied, yielding the probability of the orientations arising in the simulated recrystallization texture $f(g)^{\text {sim }}$ (Figure 1):

$$
f(g)^{\text {sim }}=f(g)^{\text {ucl }} \cdot f(g)^{\text {grow }}
$$

For that purpose, the ODFs were decomposed into a set of 936 individual orientations with various intensities $f\left(g_{i}\right)$. These 936 orientation were systematically distributed throughout the Euler space in such a way that for equal intensities, i.e. $f\left(g_{i}\right)=1$, a completely random texture results (i.e. $f(g)=1$ ) (Fortunier and Hirsch, 1987). A check of the accuracy of this decomposition procedure by re-computing the ODF according to Eq. (2) (with $\psi_{0}=5^{\circ}$ ) yielded very similar results with only minor intensity variations. To superimpose the nucleation and the growth probability, now the $f\left(g_{i}\right)$-values of the corresponding decomposed ODFs $f(g)^{\text {nucl }}$ and $f(g)^{\text {grow }}$ were multiplied. From the new data set the ODF $f(g)^{\text {sim }}$ was computed according to Eq. (2) and compared to the corresponding recrystallization textures found experimentally. This approach was applied to simulate the recrystallization textures of selected Al-alloys, where the recrystallization nucleation takes place exclusively at one distinct nucleation site (cf. next Sec.).

In most Al-alloys, however, nucleation is not restricted to solely one nucleation site, but nuclei form simultaneously at various structural heterogeneities in the microstructure. The recrystallization texture then results from a competition between the corresponding recrystallization texture components in dependence on the number and efficiency of the various nucleation sites. As already mentioned above, in heavily cold rolled commercial Al-alloys there are mainly three nucleation sites, the Cube-bands, the grain boundaries and the large particles. Therefore, for a simulation of the recrystallization textures the contribution of the three nucleation sites must be taken into account, which was conducted in the present paper by a weighted overlapping of the respective nucleation probabilities according to:

$$
f(g)^{n u c l}=x_{C u b e} \cdot f(g)_{C u b e}^{n u c l}+x_{G B} \cdot f(g)_{G B}^{n u c l}+x_{P S N} \cdot f(g)_{P S N}^{n u c l}
$$

where the probability functions $f(g)_{C u b e}^{n u c l}, f(g)_{G B}^{n u c l}$ and $f(g)_{P S N}^{n u c l}$ denote the orientation spectra provided at the Cube-bands, grain boundaries and particles, respectively, and the weight factors $x_{i}$ denote the corresponding efficiency of nucleation (with $x_{C u b e}+x_{G B}+x_{P S N}=1$ ). 
It must be mentioned here that the models applied yield no information on the evolution of the recrystallization texture or on the recrystallization kinetics. However, from comparison of the simulated textures with the experimental ones viable conclusions on the validity of the above assumptions on the interpretation of recrystallization textures by a growth selection out of a limited spectrum of preferentially formed nucleus orientations can be drawn. Furthermore, in the case of several nucleation sites being active simultaneously, the model is able to provide information on the efficiency of either nucleation site with regard to the final recrystallization texture.

\section{RESULTS OF THE SIMULATION AND COMPARISON WITH EXPERIMENTAL RECRYSTALLIZATION TEXTURES}

\section{Particle stimulated nucleation (PSN)}

In samples containing large particles $(>1 \mu \mathrm{m})$, nucleation of recrystallization takes place in the deformation zones that form around these large particles during deformation (Humphreys, 1977). With regard to the local orientations of the deformation zones and, consequently, of the resulting nucleus orientations, in single crystal experiments $\langle 112\rangle$ rotations of the matrix orientations prevail, which is attributed to the particle rotation caused by the main slip systems (Russell and Ashby, 1970, Humphreys, 1977, Engler, 1996). In polycrystalline materials, however, mostly a random orientation distribution of the recovered deformation zones is reported (Humphreys, 1977, Oscarsson, 1991), which can be interpreted by the fact that several slip systems are active simultaneously so that no defined $<112>$-rotations can result. Hence, though more detailed investigations proved that also in polycrystals the rotations can be related to the operating slip systems in a manner similar to that found in single crystals (Humphreys and Kalu, 1990, Engler, Yang and Kong, 1996), the particle-induced misorientations and the corresponding orientation distribution of recrystallization nuclei can be considered to be very close to random.

During the very early stages of annealing, mainly nuclei with a $40^{\circ}<111>$ orientation relationship grow out of the deformation zones into the deformed matrix, a process which is referred to as "micro growth selection" (Engler, Yang and Kong, 1996, Engler, 1996). In the macrotextures of the samples where recrystallization is dominated by PSN typically weak recrystallization textures with a high fraction of randomly oriented grains but also with a characteristic rotation of the Cube-orientation $\{001\}<100>$ about the ND*) towards $\{001\}<310>$ and a weak P-orientation $\{011\}<122>$ are obtained. As an example, Figure 2a shows the recrystallization texture of an $\mathrm{Al}-0.35 \% \mathrm{Fe}-0.7 \% \mathrm{Si}$ alloy pre-annealed in such a way that large particles were precipitated prior to deformation (Lücke and Engler, 1990, 1992).

In such a case of a nearly random distribution of nucleus orientation it holds $f(g)^{\text {nucl }}$ $=1$ and, hence, it is reasonable to apply the transformation ODF as discussed in the previous Sec. to take the $40^{\circ}<111>$ micro growth selection into account. Comparison of the recrystallization texture shown in Figure $2 \mathrm{a}$ with the corresponding transformed

*) Rotations of the Cube-orientation about RD- $\{h k 0\}<001>$ and TD- $\{h k 0\}<k \bar{h} 0>$ are found along the angle $\phi$ at $\varphi_{1}=0^{\circ}$ and $\varphi_{1}=90^{\circ}$, respectively, in the $\varphi_{2}=0^{\circ}$ section of the ODFs. ND-rotations $\{001\}\left\langle u \nu 0>\right.$ are visible along $\varphi_{1}$ at $\varphi_{2}=\phi=0^{\circ}$. 


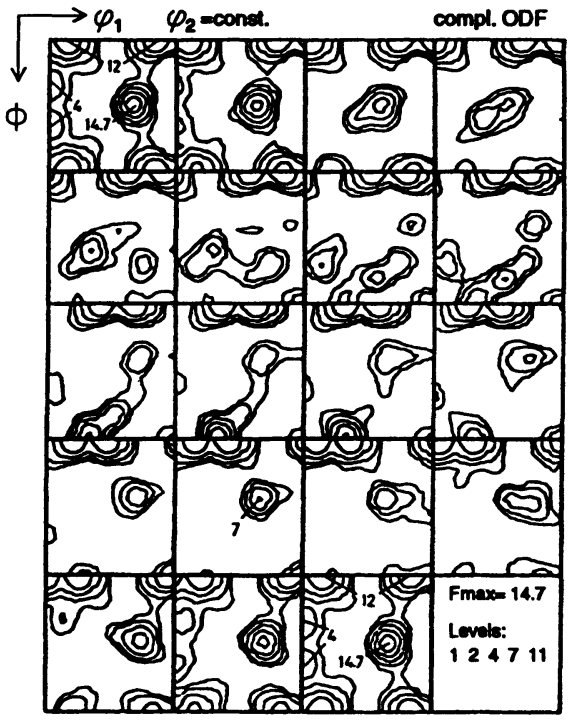

(a) Recrystallization texture (annealed for $10^{4} \mathrm{~s}$ at $300^{\circ} \mathrm{C}, \mathrm{X}$-ray)

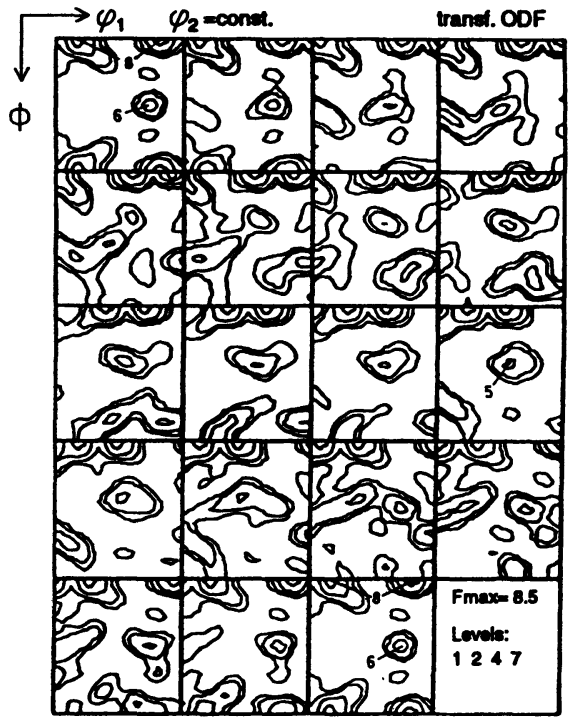

(b) $40^{\circ}<111>$ transformed rolling texture

Figure 2 Simulation of PSN (Al-0.35\%Fe- $0.7 \% \mathrm{Si}$, 95\% cold rolled).

rolling texture (Figure $2 b$ ) indeed reveals a very good agreement. In particular, the shift of the Cube peak in $\varphi_{1}$-direction (i.e. rotation about ND) as well as the occurrence of the P-orientation is very well reproduced; even the texture intensities are similar.

It should be noted that in this example a very strong rolling texture was present $(f(g) \approx 40)$ which obviously led to a strong transformation texture as well. In commercial Al-alloys, in contrast, the rolling textures are generally much weaker which is due to the larger content of particles. In that case, the resulting transformed rolling texture is very weak, too. In literature, such recrystallization textures are often referred to as "random" which, of course, is misleading. Figure 3 shows an example of a commercial strip-cast $\mathrm{Al}-1.8 \% \mathrm{Fe}-1.1 \% \mathrm{Si}$ alloy rolled to $96 \%$ reduction (Lücke and Engler, 1992). Here, a very large volume of constituent particles was present which led to a quite weak rolling texture. The resulting recrystallization ODF (Figure 3a) is also very weak, but nevertheless, it resembles the corresponding transformed rolling ODF (Figure 3b).

\section{Nucleation at Cube-bands}

The recrystallization textures of most Al-alloys are dominated by a very strong Cubeorientation $\{001\}<100>$ with strong scattering about RD*) towards Goss (e.g. Figure 4a).

*) Rotations of the Cube-orientation about RD- $\{h k 0\}<001>$ and TD- $\{h k 0\}<k \bar{h} 0>$ are found along the angle $\phi$ at $\varphi_{1}=0^{\circ}$ and $\varphi_{1}=90^{\circ}$, respectively, in the $\varphi_{2}=0^{\circ}$ section of the ODFs. ND-rotations $\{001\}<u \nu 0>$ are visible along $\varphi_{1}$ at $\varphi_{2}=\phi=0^{\circ}$. 


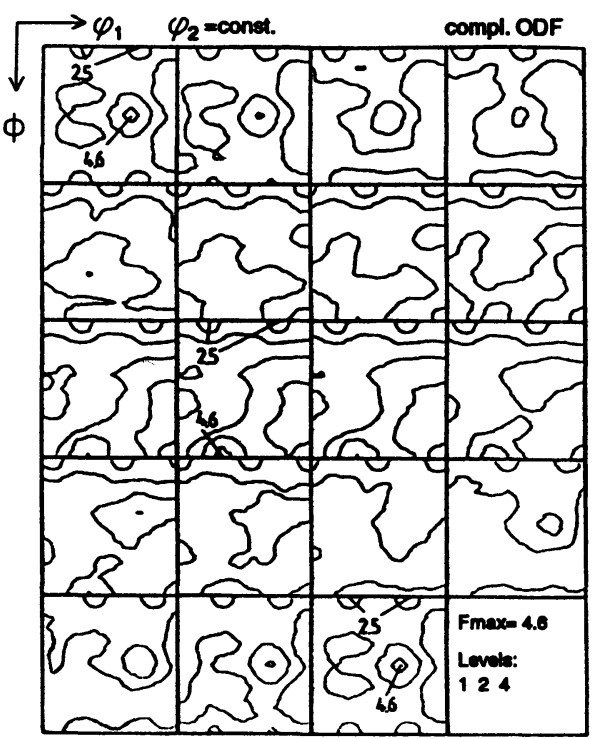

(a) Recrystallization texture (96\% red., annealed

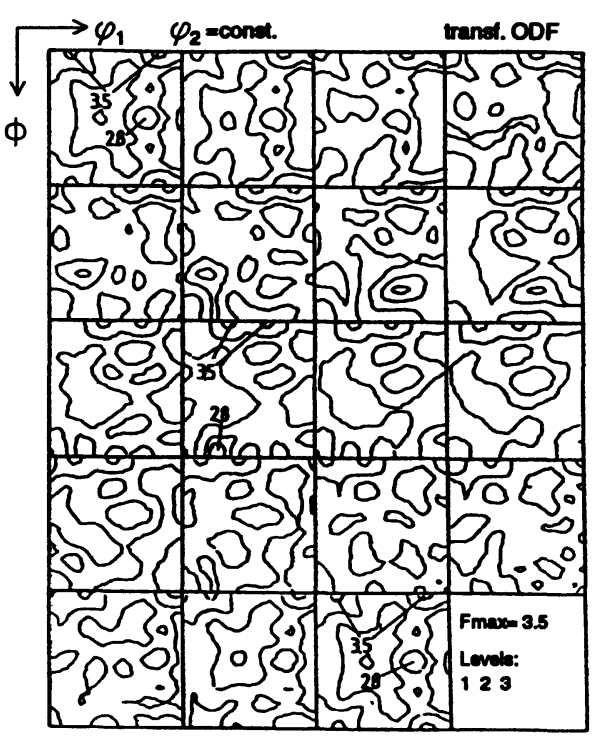

(b) $40^{\circ}<111>$ transformed rolling texture

Figure 3 Simulation of PSN (Al-1.8\%Fe-1.1\%Si, 96\% cold rolled).

Nucleation of Cube-oriented grains takes place in band-like structures, the so called Cube-bands, which are part of the deformed microstructure (either transition bands or deformed grains with retained Cube-orientation) (e.g. Hjelen, Ørsund and Nes, 1991, Weiland and Hirsch, 1991, Doherty, Kashyap and Panchanadeeswaran, 1993). In order to determine the spectrum of nucleus orientations $f(g)^{\text {nucl }}$ within the Cube-bands, the orientations of the first recrystallized grains were analysed by EBSD single grain orientation measurements in a partially recrystallized supersaturated $\mathrm{Al}-1.3 \% \mathrm{Mn}$ alloy after $97 \%$ cold rolling (Engler, Yang and Kong, 1996). Figure 4b shows an ODF which was computed from about 200 new grains that have successfully nucleated at the Cubebands. It appears that the orientations of the new grains depict strong rotations from the exact Cube-orientation either around the RD or, less pronounced, about the TD*). After complete recrystallization, however, the texture maximum is typically situated at the exact Cube-position, i.e. at $\left(0^{\circ}, 0^{\circ}, 0^{\circ}\right)$, and only some RD- and minor TD-scatter remains (e.g. Figure 4a). Thus, with progressing recrystallization an orientation shift from the rotated Cube-orientations to it exact position must take place. This means that a growth selection of the grains with an exact Cube-orientation which are present in the spectrum of nucleus orientations, presumably in the inner parts of the Cubebands, occurs. The Cube-orientation has a $40^{\circ}<111>$ orientation relationship to the Sorientation, the main texture component of the rolling textures in most cold rolled Alalloys. Furthermore, due to the high symmetry of the exact Cube-orientation such grains have favourable growth conditions into all symmetrically equivalent components of the deformation texture between $C$ and $S$ along the $\beta$-fibre. Therefore, in the long run, the exactly oriented Cube-grains grow faster than the rotated ones, forming a so called compromise texture (Juul Jensen, Hansen and Humphreys, 1985, Engler, Yang and Kong, 1996). 


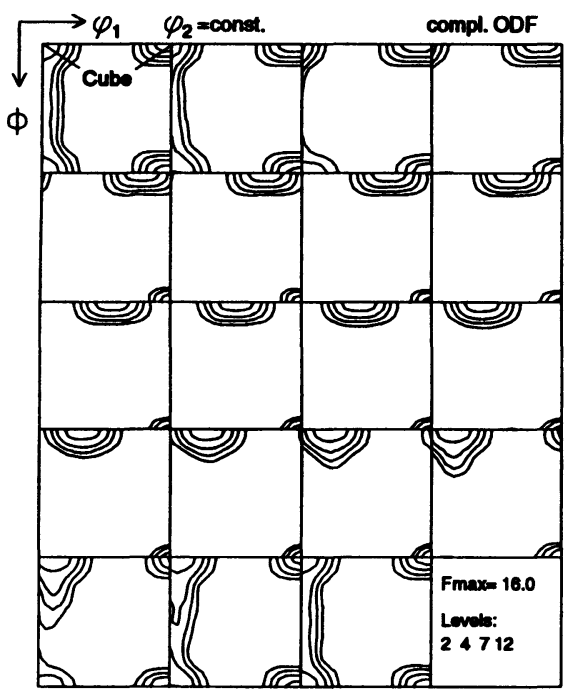

(a) Recrystallization texture (annealed for $30 \mathrm{~s}$ at $450^{\circ} \mathrm{C}, \mathrm{X}$-ray)

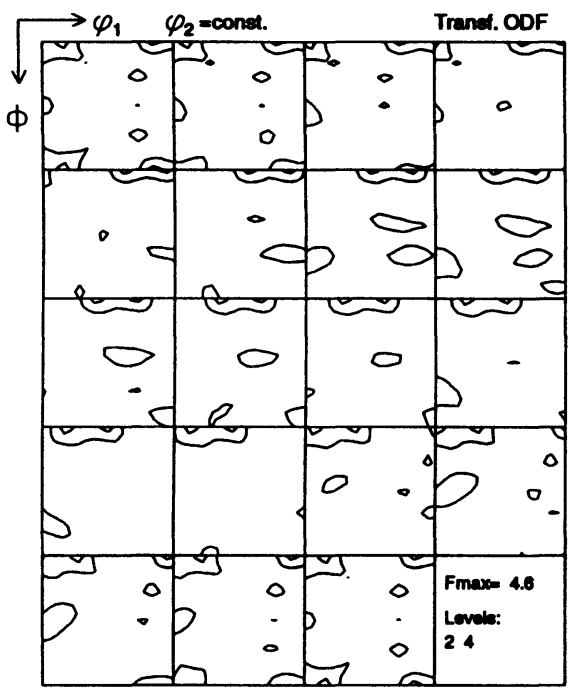

(c) $40^{\circ}<111>$ transformed rolling texture

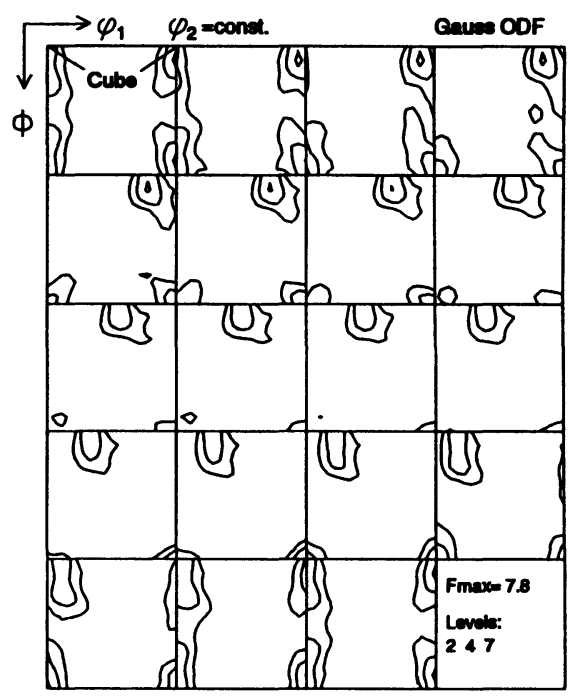

(b) ODF of the nucleus orientations (EBSD)

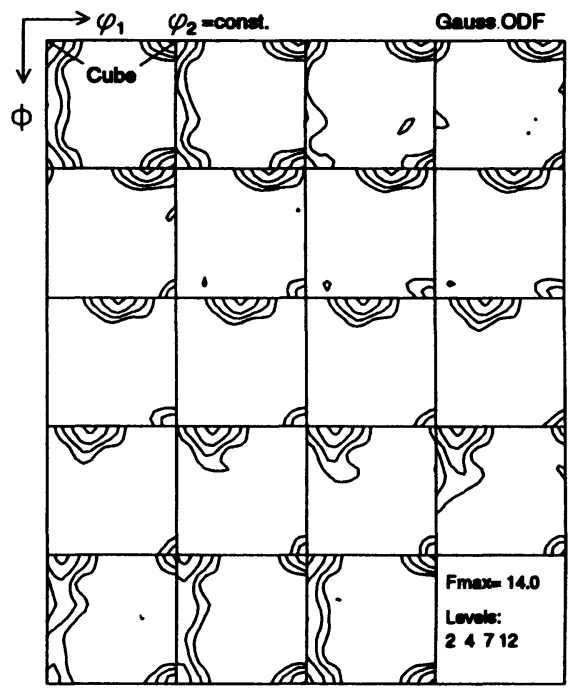

(d) Simulated recrystallization texture

Figure 4 Simulation of the Cube-recrystallization texture (Al-1.3\%Mn, 97\% cold rolled).

Based on these assumptions the recrystallization texture was simulated by superimposing the ODF of the nucleus orientations (Figure 4b) and the ODF of the corresponding rolling texture after the $40^{\circ}<111>$-transformation (Figure $4 \mathrm{c}$ ). The resulting ODF $f(g)^{\text {sim }}$ is shown in Figure 4d. Comparison of this simulated texture with that determined experimentally (Figure 4a) depicts an excellent agreement, evidencing the validity of 
the above assumptions of a $40^{\circ}<111>$ growth selection of grains with an exact Cubeorientation out of a nucleus spectrum which mainly consists of strongly scattered Cubeorientations.

\section{Nucleation at grain boundaries}

Besides the Cube-bands and the particles, the grain boundaries between the deformed grains are favourable nucleation sites in cold rolled Al-alloys. In the regions close to the grain boundaries generally higher dislocation densities and stronger orientation gradients exist due to dislocation pile-ups and the activation of additional slip systems to reduce strain incompatibilities during deformation. Therefore, best conditions for recovery and, consequently, for successful nucleation events prevail in regions close to the grain boundaries. Furthermore, the already existing large angle grain boundary between the two neighbouring grains ensures a good prospect of the nuclei. Nucleation at grain boundaries proceeds by growth of subgrains on one side of a pre-existing grain boundary into the deformed matrix on the other side beyond this boundary. Thus, this mechanism which is generally referred to as strain induced boundary migration (SIBM) leads to the formation of grains with orientations close to those present in the deformation texture (Bellier and Doherty, 1977, Doherty, 1978, Engler, 1996). Figure 5a shows an ODF $f(g)^{\text {nucl }}$ that was computed from $\sim 200$ SIBM-nuclei or new recrystallized grains which have nucleated at the grain boundaries in commercial purity Al (AA1145) after $86 \%$ cold rolling. The texture strongly resembles the rolling texture of the sample as determined by X-ray macrotexture analysis (Figure $5 b$ ), but it reveals fairly large orientation scatter which reflects the orientation gradients in the vicinity of the grain boundaries.

The recrystallization textures of many Al-alloys indeed comprise orientations similar to the rolling texture S-orientation $\{123\}<634>$, which after recrystallization are usually referred to as R-orientation (Figure 5c) (e.g. Ito, Musick and Lücke, 1983, Engler, Vatne and Nes, 1996). It must however be noted that the other rolling texture orientations B $\{011\}<211>$ and $C\{112\}<111>$ are usually missing in the recrystallization textures, although they have been found in the ODF of the nucleus orientations (Figure 5a). Hence, it is concluded that with progressing recrystallization a growth selection of the $\mathrm{R}$-oriented grains to the disadvantage of the other orientations from the nucleus spectrum takes place. In comparison to the other rolling texture orientations, the R-orientation is distinguished by a preferred growth based on a $40^{\circ}<111>$-orientation relationship to each to the symmetrically equivalent components of the S-orientation in the rolling texture. So it can be assumed that subgrains with a slightly scattered S-orientation close to the former grain boundaries are able to grow very easily into neighbouring grains, which exhibit one of the symmetrically equivalent S-components.

For a simulation of this behaviour, the ODF of the SIBM-nuclei (Figure 5a) and the corresponding transformed rolling texture were multiplied yielding the simulated texture shown in Figure 5d. With regard to the R-orientation, again a very good resemblance between this simulated texture and the corresponding experimental recrystallization texture (Figure $5 \mathrm{c}$ ) is evident. In particular the orientation shift from the S-orientation in the rolling texture towards the R-orientation in the recrystallization texture is reproduced. However, despite this good simulation of the R-orientation, the comparison also yields some significant differences which mainly concern the Cubeorientation (Figure $5 \mathrm{c}$ ): Whereas the simulation virtually exclusively predicts a strong R-orientation, in the experimental recrystallization texture also a quite strong Cubecomponent appeared and the R-orientation was less pronounced. As shown in the 


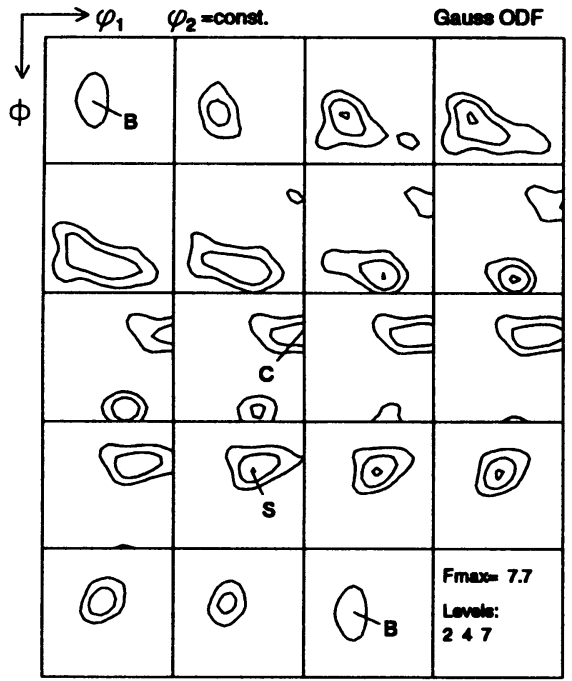

(a) ODF of the nucleus orientations (EBSD)

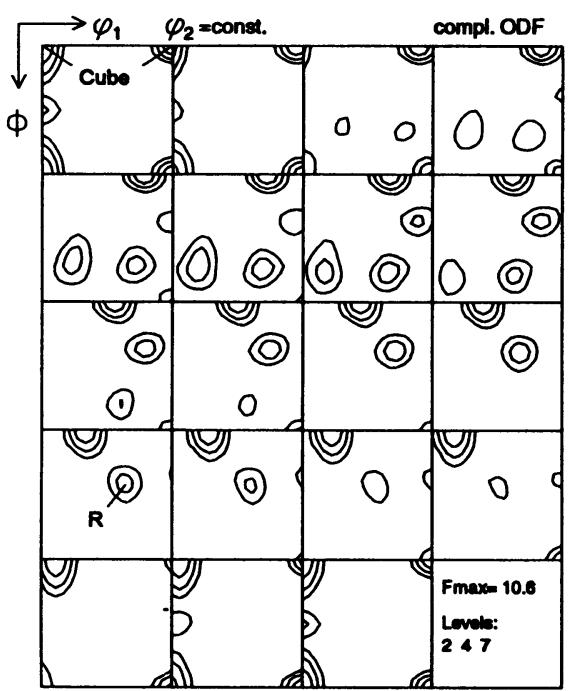

(c) Recrystallization texture (annealed for 20 s at $450^{\circ} \mathrm{C}, \mathrm{X}$-ray)

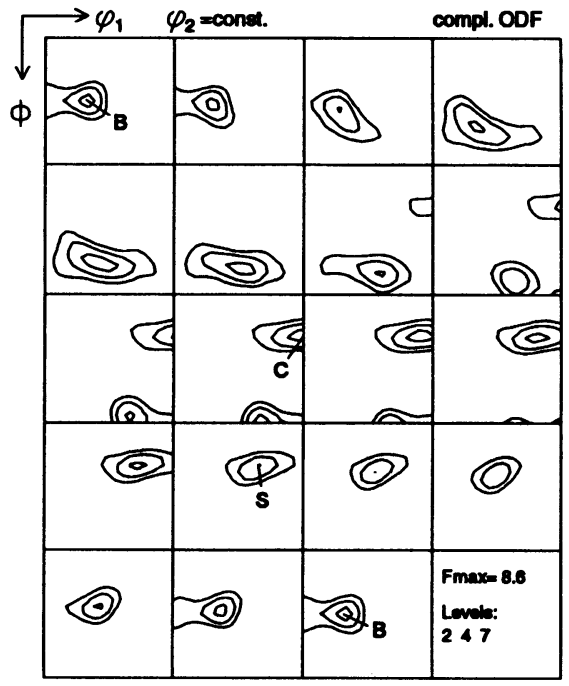

(b) Rolling texture (X-ray)

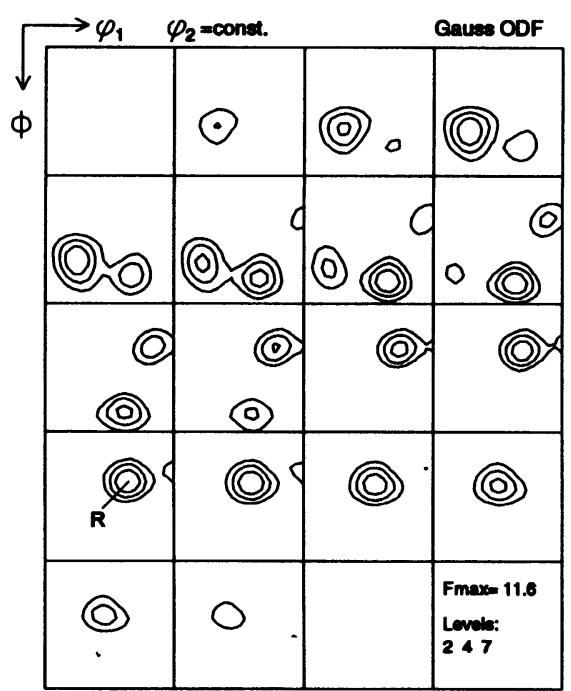

(d) Simulated recrystallization texture

Figure 5 Simulation of the recrystallization nucleation at the grain boundaries (commercial purity Al, AA1145, 86\% cold rolled).

previous Sec., the Cube-orientation is assumed to nucleate a the Cube-bands and, hence, does not occur in the orientation spectrum of the grain boundary nuclei and neither in the simulated recrystallization texture. 


\section{SIMULATION OF THE RECRYSTALLIZATION TEXTURES IN COM- MERCIAL AL-ALLOYS}

From the last example (Figure 5) it became evident that for the simulation of the recrystallization textures of most commercial Al-alloys the simultaneous nucleation at different nucleation sites must be taken into account. In the present paper, this is considered by overlapping the nucleation probabilities at the three main nucleation sites, Cube-bands, grain boundaries and particles, according to Eq. (7).

However, it is of course much too laborious to determine the potential nucleus spectrum by means of detailed EBSD-analysis in each case. Therefore, based on the orientation spectra discussed above, generalized nucleation probabilities were generated: For the nucleation of the Cube-orientation, $f(g)_{\text {Cube }}^{\text {nucl }}$ was computed by generating an ODF which consists of the Cube-orientation and its rotations up to $40^{\circ}$ about both the RD and the TD (Figure 6a; cf. Figure 4b). For the nucleation probability at grain boundaries, $f(g)_{G B}^{n u c l}$, in a first approximation the rolling texture $f(g)^{d e f}$ was taken, which gives a good hint at the frequency of the possible nucleus orientations (see Figures 5a and b). For PSN, again completely random nucleation was assumed, i.e. $f(g)_{P S N}^{n u c l}=1$.

With the help of these three nucleation probabilities, as well as with the corresponding transformed rolling textures to consider selected growth, the recrystallization textures of commercial purity Al (AA1145) were simulated. The simulation was optimized by fitting the weight factors $x_{i}$ so as to yield best agreement with the experiments, and the resulting recrystallization texture obtained with $x_{C u b e}=0.3, x_{G B}=0.5$ and $x_{P S N}=0.2$ is given in Figure $6 \mathrm{~b}$. From comparison with the recrystallization texture obtained experimentally (Figure 5c), the very good resemblance is apparent.

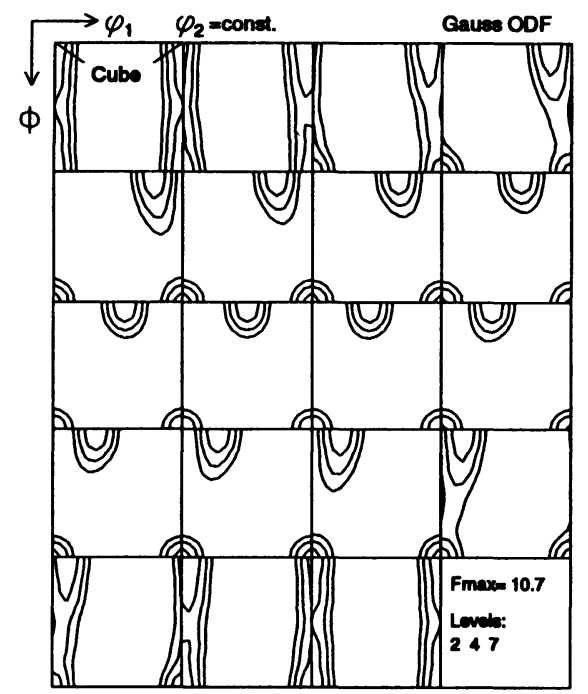

(a) Generated spectrum of nucleus orientations

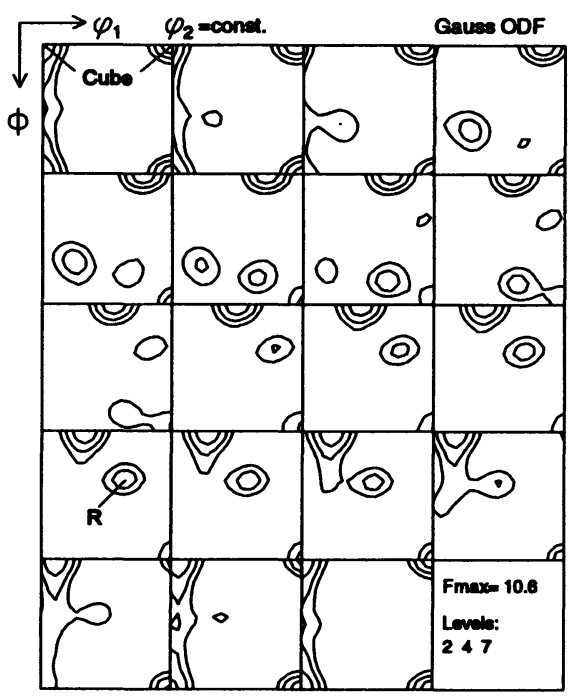

(b) Simulated recrystallization texture

Figure 6 Simulation of the recrystallization texture, taking all three types of nucleation sites into account (commercial purity $\mathrm{Al}$, AA1145, 86\% cold rolled, cf. Figure 5c). 
Thus, by means of this rather simple model recrystallization textures can readily be simulated and, in particular, it is possible to study the contribution of the different nucleation sites to the recrystallization textures. However, it must be noted that the absolute values of the weight factors $x_{i}$, and consequently the intensity of the corresponding orientations in the simulated textures, depend on the sharpness of the nucleation probability functions, so that the model is only able to provide relative data. Hence, the model is best suited to draw conclusions on the influence of various materials processings, annealing treatments, etc., on the efficiency of various nucleation sites in view of the final recrystallization textures.

\section{SUMMARY AND CONCLUSIONS}

Under the assumption that recrystallization textures of cold rolled Al-alloys form by a growth selection of $40^{\circ}<111>$-oriented grains out of a limited spectrum of preferentially formed nucleus orientations, simulation of the recrystallization textures must consider both the nucleation probability of an orientation as well as the probability of its subsequent growth. The nucleation probabilities for the most important nucleation sites in heavily cold rolled commercial Al-alloys, i.e. Cube-bands, grain boundaries and second phase particles, were derived from EBSD single grain orientation measurements. The growth probability of the various orientations was assumed to correspond to the $40^{\circ}<111>$ transformed rolling texture. Finally, the two probability functions were multiplied to simulate the recrystallization textures.

In cases where recrystallization nuclei solely form at one distinct nucleation site, the resemblance of the simulation results with the corresponding experimental recrystallization textures clearly evidenced the validity on the assumption of a growth selection out of a limited spectrum of preferentially formed nucleus orientations.

In the general case of several nucleation sites being active simultaneously, the total nucleation probability can be determined by consideration of the contribution of either nucleation site. Rather than always measuring the entire nucleation distribution by local texture analysis, the recrystallization textures can also be predicted by using artificially generated orientation spectra. This model is able to provide information on the efficiency of either nucleation site with regard to the final recrystallization texture.

\section{Acknowledgements}

Valuable discussions with Dipl.-Ing. V. Marx are gratefully acknowledged.

\section{References}

Bellier, S. P. and Doherty, R. D. (1977). Acta metall., 25, 521-538.

Bunge, H. J. (1982). Texture Analysis in Materials Science, Butterworths, London.

Bunge, H. J. and Plege, B. (1987). in Theoretical Methods of Texture Analysis, edited by H. J. Bunge, DGM-Informationsgesellschaft, Oberursel, 289-300.

Doherty, R. D. (1978). in Recrystallization of Metallic Materials, edited by F. Haeßner, Dr. Riederer Verlag, Stuttgart, 23-61.

Doherty, R. D., Kashyap, K. and Panchanadeeswaran, S. (1993). Acta metall. mater., 41, 3029-3053.

Engler, O. (1996). Mat. Sci. Tech., 12, Vol. 10.

Engler, O. and Gottstein, G. (1992). Steel research, 63, 413-418.

Engler, O., Hirsch, J. and Lücke, K. (1995). Z. Metallk., 86, 475-484.

Engler, O., Vatne, H. E. and Nes, E. (1996). Mat. Sci. Eng., A205, 187-198.

Engler, O., Yang, P. and Kong, X. W. (1996) Acta mater., 44, 3349-3369. 
Fortunier, R. and Hirsch, J. (1987). in Theoretical Methods of Texture Analysis, edited by H. J. Bunge, DGM-Informationsgesellschaft, Oberursel, 231-240.

Hjelen, J., Ørsund, R. and Nes, E. (1991). Acta metall. mater., 39, 1377-1404.

Humphreys, F. J. (1977). Acta metall., 25, 1323-1344.

Humphreys, F. J. and Kalu, P. N. (1990). Acta metall. mater., 38, 917-930.

Ito, K., Musick, R. and Lücke, K. (1983). Acta metall., 31, 2137-2149.

Jonas, J. J., Tóth, L. S. and Urabe, T. (1994). Mat. Sci. Forum, 157-162, 1713-1730.

Juul Jensen, D., Hansen, N. and Humphreys, F. J. (1985). Acta metall., 33, 2155-2162.

Köhler, U. and Bunge, H. J. (1995). Textures and Microstructures, 23, 87-114.

Köhler, U. Dahlem-Klein, E., Klein, H. and Bunge, H. J. (1992). Textures and Microstructures, 19, 125-145.

Lücke, K. (1974). Canadian Met. Quart., 13, 261-274.

Lücke, K. and Engler, O. (1990). Mat. Sci. Tech., 6, 1113-1130.

Lücke, K. and Engler, O. (1992). in Proc. 3rd Int. Conf. on Al-Alloys (ICAA 3), edited by L. Arnberg et al., The Norwegian Inst. of Tech., Trondheim, Vol. III, 439-452.

Oscarsson, A. (1991). Textures and Microstructures, 14-18, 477-482.

Pospiech, J. and Lücke, K. (1975). Acta metall., 23, 997-1007.

Pospiech, J. and Lücke, K. (1979). Z. Metallk., 70, 567-572.

Russell, K. C. and Ashby, M. F. (1970). Acta metall., 18, 891-901.

Weiland, H. and Hirsch, J. (1991). Textures and Microstructures, 14-18, 647-652. 